\title{
Methods of Accounting Aging of the Main Equipment of Nuclear Power Plants Using the Example of the Control System for the Installation of AM
}

\section{Bychok A.S., Mukhin V. Yu。, and Samokhin D. S.}

Obninsk Institute for Nuclear Power Engineering of the National Research Nuclear University MEPhl, Studgorodok 1, Obninsk, Kaluga region, 249040, Russia

\section{Abstract}

Accounting for the aging of equipment and analysis of resource characteristics of nuclear technology facilities is an urgent problem. In this paper, we show methods of solving for finding functional reliability. The analysis of the functional reliability of the most important control and protection control (SCP) systems of the AM-1 installation

Corresponding Author:

Bychok A. S

abychoks23@gmail.com

Received: 23 December 2017

Accepted: 15 January 2018

Published: 21 February 2018

Publishing services provided by Knowledge $\mathrm{E}$

(c) Bychok A. S. et al. This article is distributed under the terms of the Creative Commons

Attribution License, which permits unrestricted use and redistribution provided that the original author and source are credited.

Selection and Peer-review under the responsibility of the AtomFuture Conference Committee.

\section{G OPEN ACCESS} was also carried out.

Keywords: reliability, failure rate, mean time between failures

\section{Introduction}

The peculiarity of the installation of $A M$, as an object for evaluating the reliability indexes of its systems, is that it functioned practically without serious incidents, the entire period of its operation. The systems and components of the plant have undergone modernization during this time, some have been reconstructed, but the bulk of the circuit, layout and elemental solutions remained unchanged since the first launch. A large number of systems and elements during operation were practically not denied or refused extremely rarely, for them practically no reference data on reliability is available. they are unique. In a similar situation, the SCP system proved to be a significant number of elements that was operated from the moment the plant was commissioned to the decommissioning of the plant. Therefore, the greatest interest is in analyzing the functional reliability of the most important SCP installation systems, calculating the mean time between failures to perform certain functions. A similar calculation was carried out by Volkov Yu.V. in his work [1], but it was carried out even during the operation of the world's first nuclear power plant. In this paper, calculations were made using modern reference books on reliability, some data were refined. 


\section{Functions of SCP AM}

The most important functions of the control system are functions:

1. the formation of discrete signals of emergency protection by relative physical power and speed (period) of its change;

2. the formation of discrete signals of various stages (blocking movements, preventive protection, etc.);

3. the formation of signals in the control system for regulation and control;

4. the formation of signals about the state of systems

5. registration and presentation of information

The most important functions are the functions of enumerations 1 and 3 . These functions are used to transmit signals to the reactivity bodies. Therefore, first of all, it is necessary to calculate the reliability of SCP AM precisely for these functions.

The evaluation of the real functional reliability of the SCP AM should take into account the following circumstances:

1. Each element has its reliability

2. All elements and subsystems undergo state control, inspections, repairs, which are very likely to eliminate defects and failures

3. Not every failure of elements and systems can interfere with the performance of a particular function.

The task is to assess the actual functional reliability of the control system, taking into account all of the above circumstances, and to check whether the most important functions of the control system have been performed. The most informative is such a value as the mean time between failures. In the next chapter, evaluation techniques will be considered.

\section{Methods of solution}

The main difficulties in calculating the functional reliability of SCP AM is to take into account the regime and quality of monitoring the state of elements and systems. Methods for assessing functional reliability should begin with the already established standard methods of structural reliability.

Structural reliability of the system is the resultant reliability of the system for a given structure and known reliability values of all its parts. [2] The parts making up the system, the reliability indicators that are known, are called reliability calculation 
elements. Next, we will consider the basic methods of connecting elements to systems and the corresponding formulas for calculating the reliability of systems.

\subsection{Serial connection}

In cases where the system consists of several parts, the failure of at least one of any of which leads to the failure of the entire system, it is said that these parts are connected in series. The reliability of such a system is considered according to the formula presented below:

$$
P(t)=\prod_{i=1}^{N} P_{i}
$$

The reliability of a serial connection is always not higher than the reliability of the most unreliable element.

\subsection{Parallel connection}

In cases where the system consists of several parts and the failure of only all parts leads to a failure of the system as a whole, it is said that these parts are connected in parallel. The reliability of such a system is considered according to the formula presented below:

$$
P(t)=1-\prod_{i=1}^{N}\left(1-P_{i}(t)\right)
$$

As we see, the reliability of a parallel connection is always not lower than the reliability of the most reliable element.

To calculate the functional reliability, it is necessary to make some assumptions:

$$
\lambda=\lambda_{0}\left\{1-P_{0}\left[\operatorname { e x p } \left(-\lambda_{0} \theta+\left(1-\exp \left(-\lambda_{0} \theta\right)\left(\lambda_{0} \exp \left(-\theta \chi-\chi \exp \left(-\lambda_{0} \theta\right) /\left(\lambda_{0}-\chi\right)\right]\right\}\right.\right.\right.\right.
$$

1. An element with an intensity $x$ and an exponential law of the distribution of time between them receives a requirement to perform a given function

2. The element has exponential reliability, determined by the failure rate $\lambda_{0}$ (mean time between failures $\bar{t}_{0}=1 / \lambda_{0}$ )

3. This element undergoes periodic monitoring $\mathrm{T} \leq \mathrm{t}$ ( $\mathrm{t}$ - current time)

4. The period $\mathrm{T}=\theta+\Delta$ consists of the waiting time $\theta$ of the waiting for the service and the maximum time $\Delta \ll \theta$ 
5. Actual service time $\zeta$ is an exponentially distributed random variable with an average $\bar{\zeta}$

6. As a result of monitoring the condition and / or maintenance of the element, all faults are eliminated with probability $P_{0}$

Given all these assumptions, the intensity of dangerous failures for a given function will be:

The reliability function, with respect to dangerous failures, an element that undergoes periodic status monitoring, looks like:

$$
P(t)=e^{-\lambda t},
$$

And the average time between failures is

$$
t=1 / \lambda .
$$

where $\lambda$ is found by the formula (3).

The complexity of calculating functional reliability lies in the fact that it is necessary to take into account the operating modes of the elements. Next, two main operating modes will be considered: continuous and waiting.

\subsection{Continuous operation of the element}

The maximum operating mode of the element when the requirements are continuously $x \rightarrow \infty$, continuous operation), and the values of $\theta$ and $P_{0}$ are finite. The reliability functions of the element operating in this mode with respect to failures are found by the formula:

$$
P(t)=\exp \left\{-\lambda_{0}\left[1-P_{0} \exp \left(-\lambda_{0} \theta\right)\left(2-\exp \left(-\lambda_{0} \theta\right)\right)\right] t\right\}
$$

and the average operating time for a dangerous failure is

$$
\bar{t}=\frac{1}{\lambda_{0}\left[1-P_{0} \exp \left(-\lambda_{0} \theta\right)\left(2-\exp \left(-\lambda_{0} \theta\right)\right)\right]} .
$$

In this case, the relative increase in a mean time between failures achieved by monitoring the condition and is given by the formula:

$$
a=\frac{\bar{t}}{\bar{t}_{0}}=\frac{1}{1-P_{0} \exp (-x)(2-\exp (-x))},
$$

where $x=\lambda_{0} \theta=\theta / \bar{t}_{0}$ - is the ratio of the periodicity of the state control to the initial mean time between failures (relative periodicity). 


\subsection{Waiting for element operation}

When the requirements for the execution of a function on an element arrive at random instants of time and $x$ - is finite, it is the waiting mode of operation. If $x$ is known, then a can be estimated using formula (3). But if $X$ is unknown, we can use the following approach.

The initial reliability of the element must be chosen such that the failures of the element arise, at least, not more often than the demands on it. Hence the conclusion: if $\lambda_{0}>X$, then such an element should not be exploited. The ratio $x / \lambda_{0}=1$ is the limiting, characterizing the expediency of operating the element. For $X / \lambda_{0}=1$, it follows from formula (3) that

$$
a=\frac{1}{1-P_{0} \exp (-x)[1+(1-\exp (-x)(1+x))]}
$$

\subsection{Method for assessing the reliability of elements that were rarely rejected}

Taking into account that the element refused rarely or did not refuse at all, leads to a natural assumption about the closeness of the flow of such failures to the simplest flow [3], having the distribution of the number of events from it of the form

$$
p_{j}(t)=\frac{[\Lambda(t)]^{J}}{j !} \exp (-\Lambda(t))
$$

Where $\Lambda(t)$ is the leading function of the failure flow.

Lead function

$$
\Lambda(t)=\int_{0}^{t} \lambda(t) d t
$$

The failure flow is determined by the behavior, in time, of the danger of failures $\lambda_{t}$. If you need to take into account the aging of the element in time, then it is necessary to specify this $\lambda$-characteristic of the "aging" ", i.e. monotonically increasing in time.

Element in the course of its work passes a series of stages in which it can be exposed to natural aging, and at the end of each of them it is serviced, including for the elimination of accumulated defects.

If the number $n$ of prophylaxis is measured during time $t$ and the value is estimated $\Lambda(t)$, then, in accordance with [4], the limiting value $\lambda_{0}$ can be found from equation

$$
\Lambda(t)=\int_{0}^{\lambda_{0} t} \frac{x^{n-1} d x}{(n-1) !\left(1+x+x^{2} / 2 !+\cdots+x^{n-1} /(n-1) !\right)}
$$




\section{Evaluation of functional reliability}

When formulating the reliability of the SCP AM by function, it is important to formulate the failure of an element, device, or subsystem by function. From this, the quantitative evaluation of reliability ultimately depends. In cases where the formulation of the refusal is not obvious and can be different, one was accepted that guaranteed a minimum reliability estimate. For example, under the failure of a warning signal to exceed the power level can be understood as the failure of all 3 channels at the same time, and the failure of at least one channel. In the first case, reliability is evaluated by parallel connection of channels, and in the second one by serial connection. The second version of the compound gives the most conservative estimate. Therefore, in this example it is he who is used.

The most interesting for a more detailed analysis, were selected structural schemes:

1. the formation of a discrete signal $A 3$ by relative physical power and the rate of change in power

2. signal conditioning in the control system for automatic control

Data on the reliability of the elements and subsystems of SCP AM, taken in the required volume from [1], which are the result of either the collection and analysis of literary data or calculations performed by the authors themselves. The data were verified using modern information sources and recalculated using modern reference books and the methods listed above. of course, when drawing up the table, special attention was paid to the reliability and consistency of the data included in it.

Also in the work of Yu.V. Volkov made estimates of the missing indicators of reliability of elements based on the results of operation.

\section{The received data}

For the SCP AM system, the logic " 2 of $n$ " was chosen, according to which it is assumed that the subsystem will perform its function if at least 2 devices of the same type are in working order. In this case, it is convenient to use the Bernoulli scheme with $n$ experiments. The reliability of the function in this case is

$$
P=\sum_{m=2}^{n} C_{n}^{m} p^{m}(1-p)^{n-m}=1-(1-p)^{n}-n p(1-p)^{n-1}
$$


where $C_{n}^{m}$ is the number of combinations from $n$ to $\mathrm{m}$. Presenting the probability of "success" as

$$
p=e^{-\lambda t}
$$

where $\lambda$ is the failure flow parameter of the most unreliable device, for the reliability of a subsystem of $n$ same type elements by function, we obtain the formula

$$
P(t)=1-(1-\exp (-\lambda t))^{n-1}(1+(n-1) \exp (-\lambda t))
$$

Then for the mean time between failures, we have

$$
\bar{t}=\int_{0}^{\infty} P(t) d t=\frac{1}{\lambda} \sum_{k=2}^{n} \frac{1}{k}
$$

Using the calculation techniques listed above, we find the average time to failure for the systems of interest to us. The data obtained are presented in Table 3.

TABLE 1: Obtained data.

\section{Scheme}

Formation of the discrete signal A3 by relative physical power

Formation of the discrete signal $\mathrm{A} 3$ by relative physical power

Forming of a signal in the control system for automatic control
Mean time between failures, $\mathrm{h}$

$11,2 * 10^{5}$

$15^{\star} 10^{5}$

$2^{*} 10^{5}$

\section{Conclusions}

In this paper, the basic methods for calculating the functional reliability were presented, using the example of a control system for an AM installation. Verification calculations of the failure rate of the elements were carried out. The data obtained by the mean time between failures showed that the systems met all the reliability requirements and could perform their functions longer than the expected service life.

\section{References}

[1] Volkov Yu.V. Ocenka funkcionalnoi nadejnosti SUZ ustanovki AM _ otchet o NIR _zaklyuch., / Yu.V. Volkov__A.V. Antonov_ B.JI. Mironovich_ N.B. Saenko / IATE; Obninsk_1994._53 S 
[2] Volkov Yu.V._Duginov O.B._Klinov D.A. Nadejnost i bezopasnost YaEU_Uchebnoe posobie po kursu "Nadejnost i bezopasnost YaEU". _ 2_e izd._ pererab. i dop. Obninsk_IATE_2005 g._120 s.

[3] Klemin A.I. Nadejnost yadernih energeticheskih ustanovok. Osnovi rascheta. M. Energoatomizdat_ $1987 \mathrm{~g}$.

[4] Volkov B.V._ Chistozvonova E.A. Ob ocenke nadejnosti redko otkazivayuschih elementov YaEU. Izv. VUZov. Yadernaya energetika №1_ $1993 \mathrm{~g}$. 